\title{
Brillouin Effect Impact in RoF Systems with Photonic-Assisted RF Amplification
}

\author{
E. S. Lima, L. A. M. Pereira, R. M. Borges, M. S. B. Cunha and Arismar Cerqueira S. Jr.
}

\begin{abstract}
We investigated the impact of the stimulated Brillouin scattering (SBS) in radio over fiber (RoF) system with photonic-assisted radiofrequency (RF) amplification. Such photonically amplified RoF systems employ long pieces of highly nonlinear fibers (HNLFs) for stimulating the four-wave mixing (FWM) nonlinear effect, in conjunction with appropriate management of the optical modulation index, aiming to provide RF gain. Numerical and experimental results demonstrate SBS might reduce the FWM nonlinear efficiency and limit the overall system power efficiency. We propose using only $35 \mathrm{~m}$ of HNLF for ensuring high photonic-assisted RF gain and increasing the SBS threshold in $14 \mathrm{~dB}$. The digital performance of the photonically amplified RoF system is investigated in terms of root mean square error vector magnitude (EVM $\mathbf{R M S}_{\text {S }}$, before and after mitigating SBS. The 35-m long HNLF piece implies in enhancing the system digital performance and reducing in $6 \mathrm{~dB}$ the required optical power for attaining $19 \mathrm{~dB}$ of photonic-assisted RF gain.
\end{abstract}

Index Terms-Four-wave mixing; Nonlinear optics; Radio over fiber; Stimulated Brillouin scattering.

\section{INTRODUCTION}

$\mathrm{T}$ HE growing demand for mobile communications, data exchange and coverage has stimulated the fifth-generation (5G) wireless networks development, which has become a hot topic in both industry and academia in the past few years. $5 \mathrm{G}$ is predicted to bring a transformation to the human society, dealing with tough requirements from key usage scenarios, namely: enhanced mobile broadband (eMBB); massive machine-type communications (mMTC); ultra-reliable and low latency communications (URLLC). The high mobility (up to $500 \mathrm{~km} / \mathrm{h}$ ) and extreme long-range coverage (up to $100 \mathrm{~km}$ ) use cases have also triggered a lot of interest, mainly in continentalscale countries [1]-[4]. Accordingly, the emerging 5G networks are going to take advantage of the recently launched $5 \mathrm{G}$ new

Article submitted on March, 2020. This work was supported in part by RNP, with resources from MCTIC, Grant No. 01250.075413/2018-04, under the Radiocommunication Reference Center (Centro de Referência em Radiocomunicações - CRR) project of the National Institute of Telecommunications (Instituto Nacional de Telecomunicações - Inatel), Brazil; in part by CAPES; in part by FINEP; in part by CNPq; in part by FAPEMIG. (Corresponding author: Arismar Cerqueira Sodré Junior.)

E. S. Lima, L. A. M. Pereira and Arismar Cerqueira S. Jr. are with the National Institute of Telecommunications, Santa Rita do Sapucaí, MG 37540000, Brazil (e-mail: elima@get.inatel.br; luiz_augusto@get.inatel.br; arismar@inatel.br).

R. M. Borges and M. S. B. Cunha are with the National Institute of Telecommunications, Santa Rita do Sapucaí, MG 37540-000, Brazil, and also with the Federal University of Itajubá, Itajubá, MG 37500-903, Brazil (e-mail: ramonmb@inatel.br; matheusseda@gee.inatel.br ).

Digital Object Identifier: 10.14209/jcis.2020.17 radio (NR) standard, new waveforms, additional spectral bands including millimeter-waves (mm-waves), massive multipleinput multiple-output (mMIMO) techniques and advanced antennas [5-7]. In parallel, the radio access network (RAN) should evolve to accommodate the $5 \mathrm{G}$ vision, in such a way, the physical layer network review points out to fiber-wireless convergence, heterogeneous networks (HetNets) with a mix of cell sizes, and centralized RAN (C-RAN) [8][9].

CRAN consists of keeping the remote radio unit (RRU) close to the antenna site and displacing the baseband unit (BBU) from remote cell sites to a unified central unit (CU). The C-RAN key advantages over the traditional distributed RAN (D-RAN), which physically locates BBU with RRU in a base station (BS), are the following ones: centralization of several base stations functions in a single room, enabling to share facilities as power and air-conditioning; management efficiency and reduced network deployment time, making practicable the BSs number increasement; easier and agile communication between colocated BBUs, contributing to the low latency applications and energy consumption reduction. These features contribute to reducing both capital and operational expenditures [9][10].

On C-RAN, CU can connect RRU by using a physical link, known as fronthaul (FH), or employing an optical midhaul (MH) up to a distribution unit (DU) followed by the FH, which can be optical or wireless despite it is mostly based on singlemode fiber (SMF). Along with the backhaul (BH), i.e. the link between $\mathrm{CU}$ and core network, the $\mathrm{FH}$ and $\mathrm{MH}$ transport networks compose the next generation fronthaul interface (NGFI), giving rise to the $5 \mathrm{G} \mathrm{X}$-haul vision as illustrated in Fig. 1. It integrates backhaul and fronthaul into a common physical architecture supporting transport and access for multiple technologies [9].

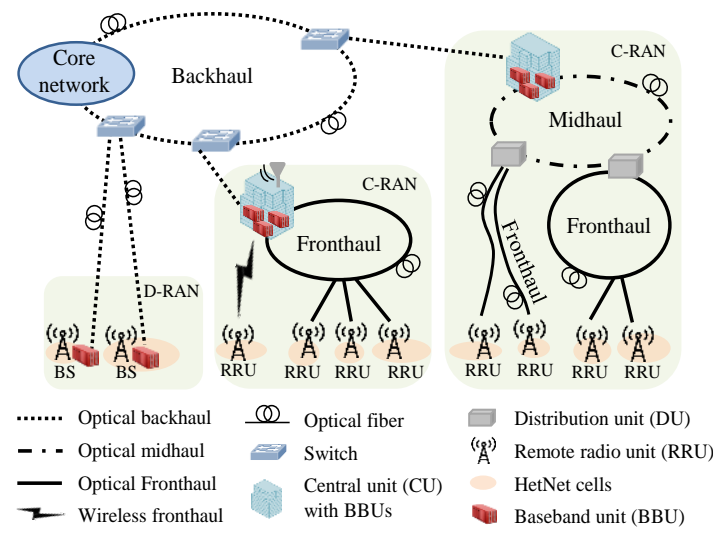

Fig. 1. The 5G X-haul vision regarding the main physical architecture. 
In this context, Microwave Photonics (MWP) has been recognized as a key area of study to simplify the network architectures, by taking advantage of optical and mobile communications in a unique heterogeneous system [11-13]. While photonics-based radiofrequency (RF) generation and processing can overcome the electronics-based hardware limitations of high-frequency wireless systems [12], radio over fiber $(\mathrm{RoF})$ technology enables high capacity fronthaul links for multiband and Gbit/s 5G communications networks [14][15]. An RoF link might be analog (A-RoF) or digital (D-RoF). Although D-RoF is the main available solution today, A-RoF topologies have been gradually becoming attractive as the industry begins to deploy mm-waves access and scalable $5 \mathrm{G}$ networks [16].

Analog RoF links can suffer from nonlinear effects, including four-wave mixing (FWM), stimulated Raman scattering (SRS) and stimulated Brillouin scattering (SBS). The two latter ones are typically avoided in an optical communication system, by not exceeding the optical power thresholds that lead to nonlinearities $\left(P_{\text {th }}\right)$ [17]. On the other hand, nonlinear optics can be properly exploited to bring benefits for optical-wireless systems, mainly in the microwaves and mm-waves bands [18][19]. Particularly, FWM has been demonstrated useful for enabling photonic-assisted RF amplification (PAA) in A-RoF systems [20-24]. PAA consists of increasing the photodetected electrical power without requiring electrical amplifiers or high-power photodetectors (PDs). Therefore, it can be applied to the backhauls and fronthauls transport networks from Fig. 1, making them an active medium for performing RF signal amplification besides data transmission and, consequently, reducing or even eliminating the requirements of the electrical amplifiers.

According to the literature, Wall and Foster reported microwave amplification in A-RoF systems using FWM and reported $9 \mathrm{~dB}$ photonics-based $\mathrm{RF}$ gain for frequencies up to $14 \mathrm{GHz}$ [20]. Our research group has intensely contributed to this topic in the last years [21-25], attaining world records in terms of maximum photonics-assisted RF gain of $27 \mathrm{~dB}$ and widest bandwidth up to $50 \mathrm{GHz}$. Particularly in [21] and [22], we have demonstrated the physical mechanism behind PAA, which not only due to the FWM effect, but also to the modulation sidebands enhancement. Afterward, the PAA technique has been improved and experimentally evaluated as a function of diverse parameters and figures of merit, as following [23][24]: pump wavelength spacing, polarization voltage $\left(V_{\text {bias }}\right)$ of a Mach-Zehnder modulator (MZM); RFdriven signal level; signal-to-noise ratio (SNR); phase noise; root mean square error vector magnitude $\left(\mathrm{EVM}_{\mathrm{RMS}}\right)$; spuriousfree dynamic range (SFDR); temperature variation. Recently, we have proposed a novel PAA approach based on a single pump, as well as the implementation of multiband and photonically amplified fiber-wireless Xhaul [25].

In all aforementioned PAA approaches, FWM has been stimulated by using highly nonlinear fibers (HNLFs) and optical pumping. However, SBS might also occur, since its threshold is typically lower than that of FWM. This might be considered a drawback, because SBS manifests through the generation of a backward-propagating Stokes wave, degrading the optical signal-to-noise ratio (OSNR) [17]. Techniques for suppressing SBS in conventional optical communications systems have been proposed in the last decades [26-28]. However, SBS has not been properly evaluated in photonically amplified RoF links so far, apart from our preliminary results that have been freshly published in a Brazilian national conference [29].

This work proposes the use of only $35 \mathrm{~m}$ of HNLF for ensuring high photonics-assisted RF gain and increasing the SBS threshold. The paper main contributions are the following ones: experimental evidence of the undesired Brillouin nonlinear effect in the PAA approach; identifying an appropriate HNLF length to mitigate SBS and ensure high photonics-assisted gain; enabling the SBS threshold increase for improving the overall system power efficiency, with enhanced digital performance in terms of EVM $\mathrm{RMS}_{\mathrm{S}}$

The manuscript is structured in five sections. Section II describes RoF systems with photonic-assisted amplification, whereas Section III reports the impact of the Brillouin effect on them. Section IV presents the numerical and experimental results of the SBS-free photonically amplified RoF systems. The conclusions and suggestions for future works are drawn in Section V.

\section{ROF SYSTEM WITH PHOTONIC-ASSISTED RF AMPLIFICATION}

In this section, we describe the photonic-assisted RF amplification technique based on RoF and FWM, which is able to provide RF gain from baseband (BB) to extremely high frequency (EHF) [22]. The photonic-assisted RF gain is defined as the difference between the photodetected electrical power for PAA (Fig. 2b) and a conventional RoF system (Fig. 2a), named as CRoF, by maintaining the same optical power level at the PD input for both approaches. The main $\mathrm{CRoF}$ components are a laser diode (LD), a Mach-Zehnder modulator, a fiber-optic link and a photodetector.

In the PAA approach, two laser diodes are properly spaced by $2.3 \mathrm{~nm}$ and set around the HNLF zero-dispersion wavelength $\left(\lambda_{0}\right)$, with the purpose of enhancing the four-wave mixing nonlinear effect. These two optical waves are copolarized by using polarization controllers (PC), and posterior combined by an optical coupler (OC) with 4-dB insertion loss. Sequentially, the optical beam goes to an MZM modulator, before being amplified by an Erbium-doped fiber amplifier (EDFA) and launched to a piece of highly nonlinear fiber. Its relevant parameters are as follows: dispersion slope at $1,550.00 \mathrm{~nm} \quad S_{0}=0.023 \quad \mathrm{ps} / \mathrm{nm}^{2} / \mathrm{km}$; length $L=470 \mathrm{~m}$; attenuation factor $\alpha=0.8 \mathrm{~dB} / \mathrm{km}$; effective area $A_{\text {eff }}=8.9 \mu \mathrm{m}^{2}$; $\lambda_{0}=1557 \mathrm{~nm}$; nonlinear coefficient $\gamma=28 \mathrm{w}^{-1} \mathrm{~km}^{-1}$. The MZM modulates both optical carriers with the RF-driven signal, provided by a vector signal generator (VSG). The bias voltage has been set between quadrature (QP) and minimum transmission point (MITP) to increase the modulation index and, consequently, the sidebands power level. Finally, EDFA 


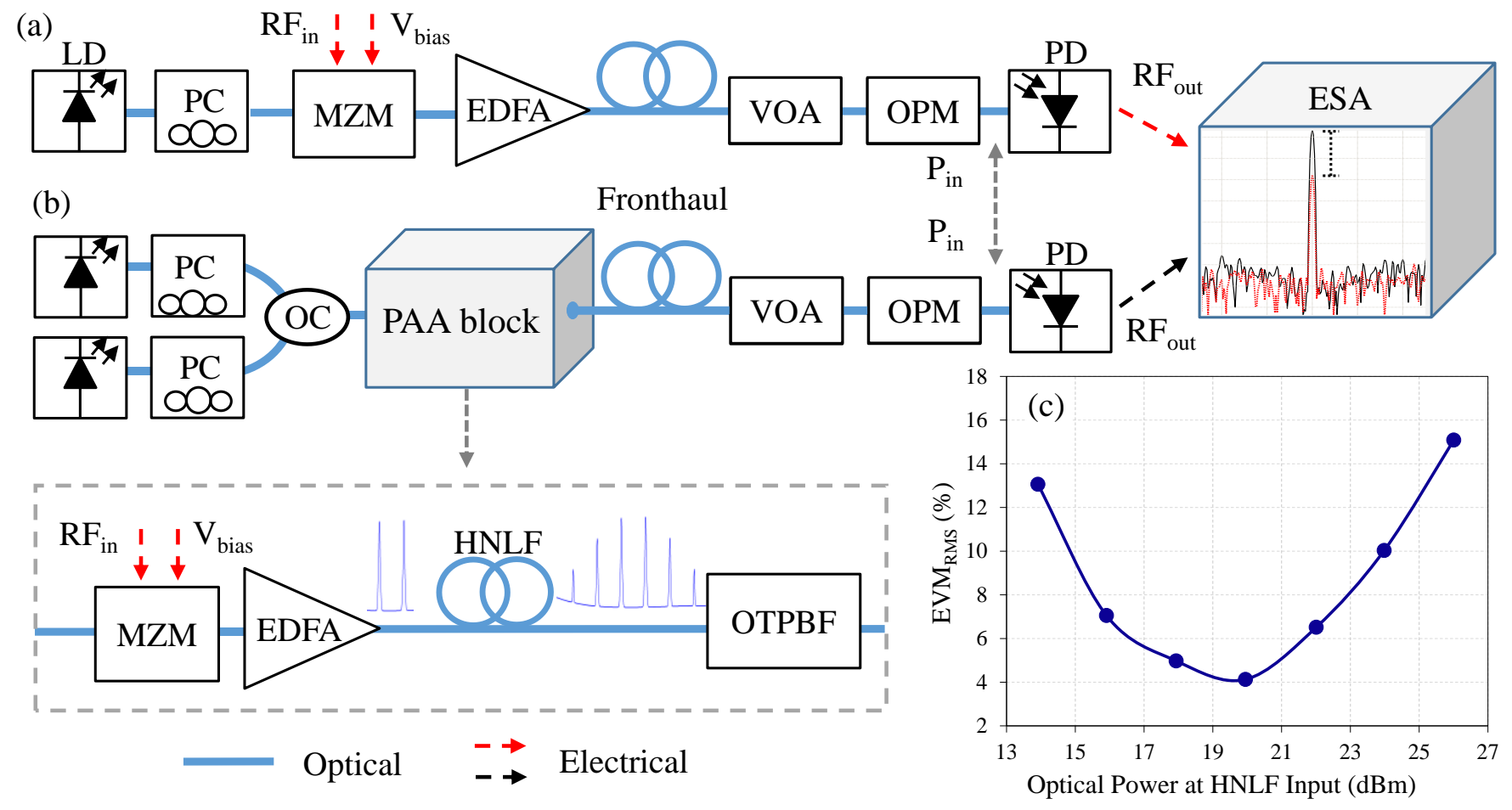

Fig. 2. (a) Conventional radio over fiber (CRoF). (b) Photonic-assisted amplification technique (PAA). (c) EVM $_{\mathrm{RMS}}$ as a function of the optical power at the 470-m HNLF input; LD - laser diode; PC - polarization controller; MZM -Mach-Zehnder modulator; EDFA - erbium-doped fiber amplifier; SMF - single mode fiber; OPM - optical power monitor; PD- photodetector; ESA - electrical spectrum analyzer; OC - optical coupler; HNLF - highly nonlinear fiber; OTPBF - optical tunable pass-band filter; VOA - variable optical attenuator.

increases the total input power for increasing the FWM nonlinear efficiency [19].

The beating between the two optical carriers produces new FWM products, as presented in the HNLF output from Fig. 2 (b). The joint effect of FWM and high modulation index gives rise to photonic-assisted RF amplification. An optical tunable bandpass filter (OTBPF) with $0.8 \mathrm{~nm}$ bandwidth selects the first left or right FWM product and launches it into a 12.5$\mathrm{km}$ optical fronthaul of single-mode fiber. A variable optical attenuator (VOA) and an optical power monitor (PM) have been used for ensuring the same optical power at the PD input for both approaches. Finally, a photodetector performs the opticalto-electrical conversion of the RF-driven signal $\left(\mathrm{RF}_{\text {in }}\right)$. An electrical spectrum analyzer (ESA) has been used for comparing the resultant RF signals from $\mathrm{CRoF}$ and PAA. A vector signal generator has been used to transmit a continuous wave (CW) at $6 \mathrm{GHz}$ with $0 \mathrm{dBm} \mathrm{RF}$ power. One can observe in ESA of Fig. 2 that the PAA approach provides higher RF power level and SNR in comparison to CRoF for the same optical power, demonstrating the photonic-assisted gain. Moreover, the PAA provides RF gain without increasing the electrical noise floor, which has been kept constant for both systems.

As mentioned in Section I, SBS has not been well investigated in photonically amplified RoF links yet, except our preliminary results reported in [29]. Indeed, the performance results from [22] proved the measured error vector magnitude is reduced as the total optical power is increased due to the photonics-assisted RF gain boost up to an inflection point around $20 \mathrm{dBm}$. This phenomenon is illustrated in Fig. 2(c), by means of reporting an experimental analysis of $\mathrm{EVM}_{\mathrm{RMS}}$ as a function of the optical power at the 470-m HNLF input. The $\mathrm{EVM}_{\mathrm{RMS}}$ parameter might be understood as a deviation from the received symbols, regarding the reference constellation symbols. Therefore, it is expected that $\mathrm{EVM}_{\mathrm{RMS}}$ would be directly proportional to HNLF, which is responsible to enhance the FWM effect. However, power levels higher than $20 \mathrm{dBm}$ decrease the system performance due to higher values of $E M_{R M S}$ as a result of a reduction on the photonic-assisted RF gain. This phenomenon is the main motivation of the current work since it can be explained by the consequences of stimulated Brillouin effect. Under this condition, the optical power is backscattered and the optical signal-to-noise ratio is reduced. A brief description of Brillouin effect and a detailed investigation on its impact in radio over fiber systems with photonic-assisted RF amplification are the focus of Section III and Section IV, respectively.

\section{SBS - STIMULATED BRILLOUIN SCATTERING}

The stimulated Brillouin scattering occurs in optical transmissions when the transmitted photons interact with acoustic phonons, generating Stokes photons. In 1964, for the first time, this effect was experimentally observed in Sapphire and Quartz crystals [30]. SBS is nonlinear since for optical powers above the Brillouin threshold $\left(P_{\mathrm{th}}\right)$, the Stokes wave exponentially growths and propagates backward of the desired propagation direction. In this way, this process decreases the 
optical power at the interested frequency, since the reflected signal optical components are shifted in accordance with the optical fiber characteristics. The Brillouin shift between the transmitted and reflected optical carriers can be defined as

$$
\Delta f=2 f_{0} \frac{V}{c / n_{1}}
$$

in which, $f_{0}$ is the original optical signal frequency, $V$ is the acoustic wave velocity, ${ }^{c} / n_{1}$ is the light group velocity into the fiber, $n_{1}$ and $c$ are the core refractive index and light velocity in vacuum, respectively. In general, the Brillouin shift comprises frequencies from 9 and $11 \mathrm{GHz}$ [17]. SMF typically presents Brillouin shift around to $11 \mathrm{GHz}$, whereas $9 \mathrm{GHz}$ is expected for optical fibers with a dense concentration of Germanium $\left(\mathrm{GeO}_{2}\right)$ [17].

The Stokes wave propagates into the backward direction of the original signal direction. Hence, SBS can be used for amplifying signals transmitted in the same optical fiber, frequency, and direction of the Stokes wave. However, the narrowband spectrum of the reflected signal limits its application as an optical amplifier. On the other side, the SBS effect limits the maximum transmission optical power, which must be lower than the Brillouin threshold defined as [17]

$$
P_{t h} \approx \frac{21 \times A_{\mathrm{eff}}}{g_{\mathrm{B}}\left(V_{\mathrm{B}}\right) \times L_{\mathrm{eff}}}
$$

in which, $A_{\text {eff }}$ is the effective mode area, $g_{\mathrm{B}}\left(V_{\mathrm{B}}\right)$ is the Brillouin coefficient gain (from 3 to $5 \times 10^{-11}$ ) $\mathrm{m} / \mathrm{W}$ and $L_{\text {eff }}$ is the optical fiber effective length. The last one depends on the link length $(L)$, the attenuation factor $(\alpha)$ and is given by [17]

$$
L_{\mathrm{eff}}=\frac{1-e^{-\alpha L}}{\alpha}
$$

The nonlinear Brillouin effect is inherent to the optical communications and becomes more significant for long-range transmissions. There are some techniques for measuring and suppressing SBS. Fig. 3 (a) illustrates the block diagram of the technique used for measuring the SBS shift in this paper [31]. A laser diode has generated the optical carrier and a polarization controller has controlled its polarization, before being launched into the optical fiber. An EDFA has amplified the optical signal, increasing the power density at the HNLF core for enhancing nonlinear effects. The amplified optical carrier has been applied to an optical splitter $(1 \times 2)$, which has divided the original signal in two components (i) and (ii). Part of the signal (i) has been applied to the optical circulator and then, launched into the HNLF, which favors nonlinear effects due to the HNLF high nonlinearity coefficient $\left(\gamma=28 \mathrm{~W}^{-1} \mathrm{~km}^{-1}\right)$. A VOA and a power monitor have been used for controlling and monitoring the optical signal, respectively. The transmitted signal has been attenuated in $20 \mathrm{~dB}$ in order to ensure the maximum optical power level supported by the power monitor. An optical coupler
$(2 \times 1)$ has combined the reflected Brillouin signal (iii) and part of the original wavelength (ii). The VOA has been used for ensuring a safe optical power for the photodetector $(10 \mathrm{dBm})$. Fig. 3 (b) illustrates the SBS gain spectrum, which has been generated using the heterodyne method. One can note the power spectral density centralized at $9.1 \mathrm{GHz}$, which refers to the measured Brillouin shift at HNLF.
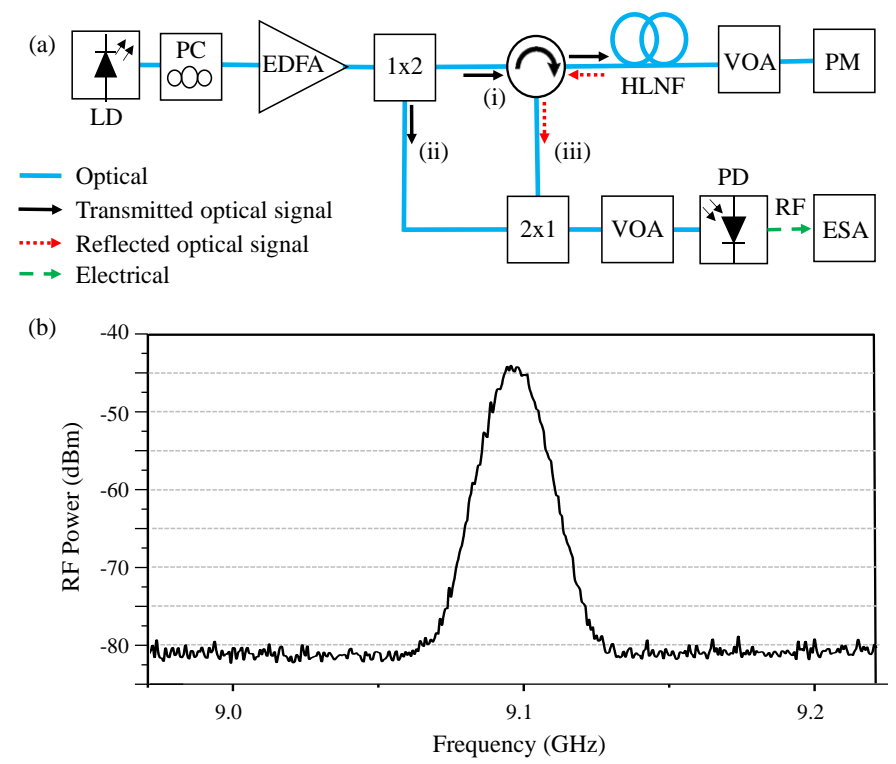

Fig. 3. Stimulated Brillouin Scattering measurement: (a) Experimental setup; (b) Measured Brillouin gain spectrum.

\section{SBS INVESTIGATION IN RoF SYSTEMS WITH PHOTONIC- ASSISTED RF AMPLIFICATION}

This section is regarding an experimental investigation of the stimulated Brillouin scattering in the proposed RoF systems with photonic-assisted RF amplification. It has been divided in two subsections, namely: Brillouin effect impact and reduction; digital analysis using an appropriate HNLF length.

\section{A) Brillouin Effect Impact and Reduction}

Overcoming the Brillouin optical power threshold, defined by Eq. (2), implies in causing transmitted power saturation and exponential reflected power increasement. An experimental investigation has been performed by using $470 \mathrm{~m}$ of HNLF in order to validate its value and obtaining the point, in which the reflected power reaches the transmitted power level. Fig. 4 illustrates the experimental setup for this characterization. A laser centralized around the HNLF $\lambda_{0}(1,557.35 \mathrm{~nm})$ has the polarization adjusted by a PC and the optical power amplified

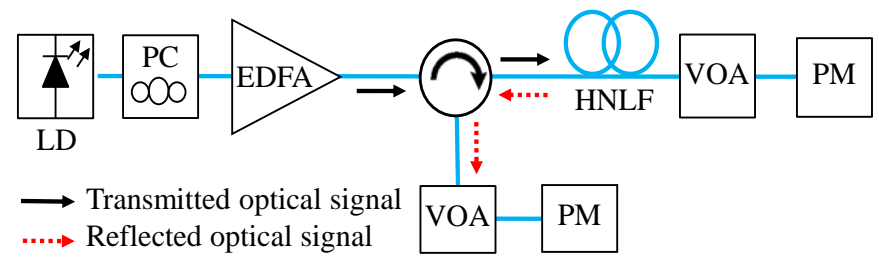

Fig. 4. Block diagram for measuring Brillouin threshold in the HNLF. 
by an EDFA with tunable gain. The amplified signal has been inserted in an optical circulator, which has the transmission port connected at the HNLF input and the reflection port coupled in a VOA and a PM, sequentially. Similarly, the transmitted power throughout the fiber passes through a second VOA and has been measured in another power monitor.

Fig. 5 presents the transmitted and reflected power curves, as a function of the optical power at the HNLF input. The Brillouin power threshold can be observed when optical power in HNLF is around $15 \mathrm{dBm}$. One can note a saturation of the transmission power and an exponential increase of the reflected power. The curves intersection point occurs when the optical power at HNLF is $21 \mathrm{dBm}$. It is observed the transmitted power remains constant, whereas the reflected power increase due to the SBS effect. Therefore, as presented in Fig. 2 (c), reduced system performance is expected for power levels higher than $21 \mathrm{dBm}$, as a consequence of the Brillouin reflected optical power.

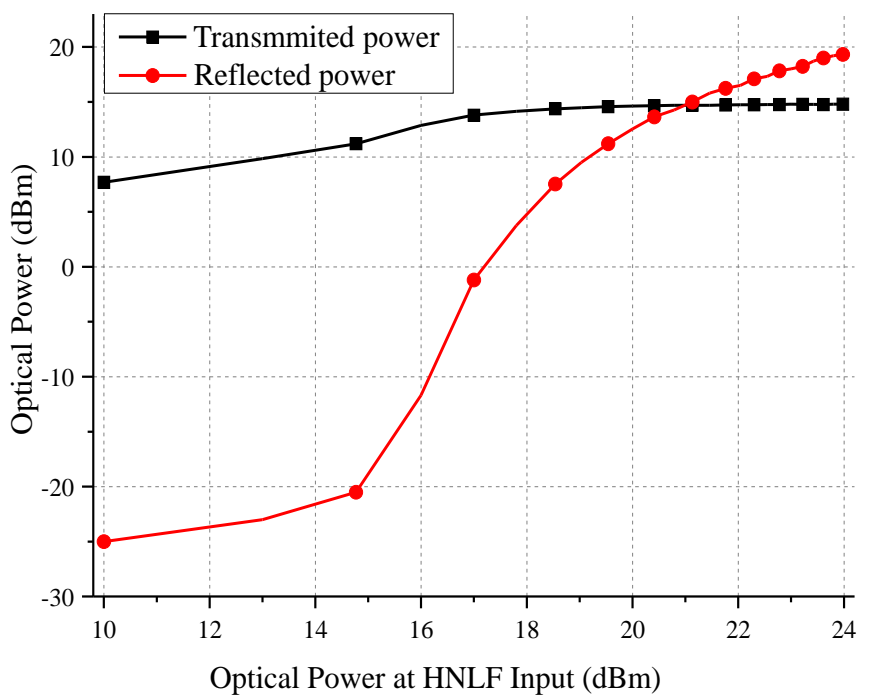

Fig. 5. Brillouin effect experimental analysis as a function of the optical input power for $470 \mathrm{~m}$ of HNLF.

A numerical analysis has been realized using Optisystem for comparison purposes between the experimental and simulated results. We have elaborated a reliable simulation model for analyzing the reflected and transmitted optical power levels, as a function of the HNLF length. Thus, we could reproduce the experimental results reported in Fig. 5 and, consequently, calibrating and validating our numerical simulations. This research stage has considered all HNLF characteristics, and the obtained numerical curves are presented in Fig. 6. One can observe the simulated curves are in accordance with the experimental results since the interception between the curves occurs around $21 \mathrm{dBm}$ for the experimental results and approximately $20 \mathrm{dBm}$ for the numerical.

Complementary, a second numerical investigation has been realized, aiming to increase the Brillouin effect power threshold and consequently, reducing its impact on the performance of the photonically-amplified RoF system. The HNLF effective length has been considered as the parameter to be managed. In long links, the $L_{\text {eff }}$ can be approximated as the reverse of the attenuation factor, and in shorts distances $(\alpha L \ll 1)$, the $L_{\text {eff }}$ is the fiber physical length. Therefore, the HNLF length reduction implies on increasing the SBS threshold. The numerical investigation considered the relevant HNLF parameters and the optical power at the HNLF input has been set to $20 \mathrm{dBm}$, which presents the optimal EVM RMs point in accordance to our previous PAA experiments.

Fig. 7 displays the transmitted and reflected optical power curves as a function of the HNLF length from 1 to $470 \mathrm{~m}$. One can observe the transmitted power decreases as the HNLF length is increased. Particularly, when the HNLF length reaches $470 \mathrm{~m}$, the reflected power is approximately $10 \mathrm{~dB}$ above the transmitted power. The zoom-in-view of Fig. 7 shows the intersection point between the curves, which occurs around $54 \mathrm{~m}$ of HNLF. Therefore, we have decided to separate our HNLF spool into two pieces, with the purpose of minimizing the Brillouin effect and intensifying the FWM generation efficiency. The chosen HNLF length was approximately $35 \mathrm{~m}$, resulting in a transmitted power level above the reflected power, intending to apply a trade-off between reducing the

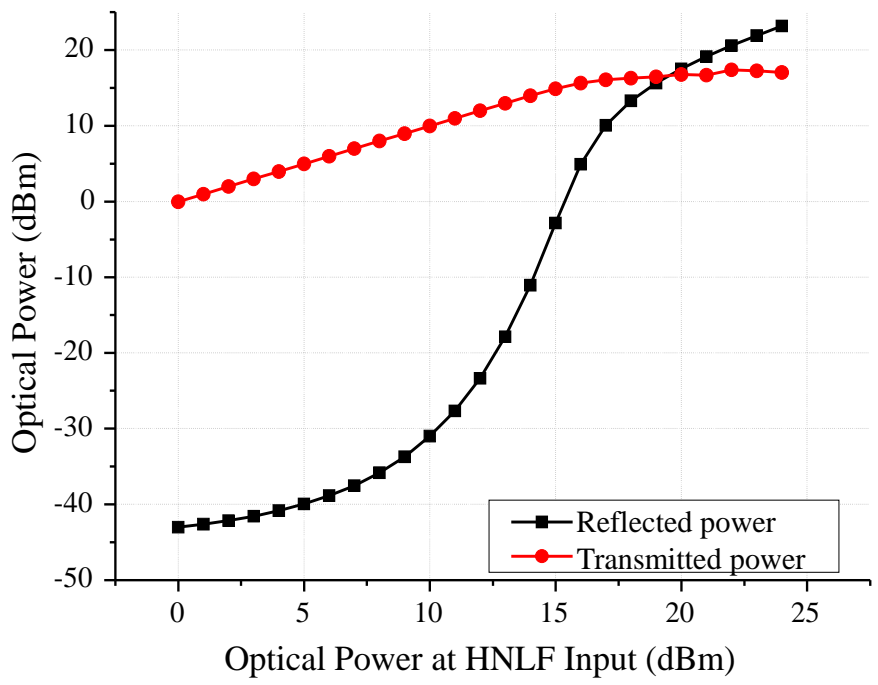

Fig. 6. Numerical analysis of the Brillouin effect as a function of the optical input power for $470 \mathrm{~m}$ of HNLF.

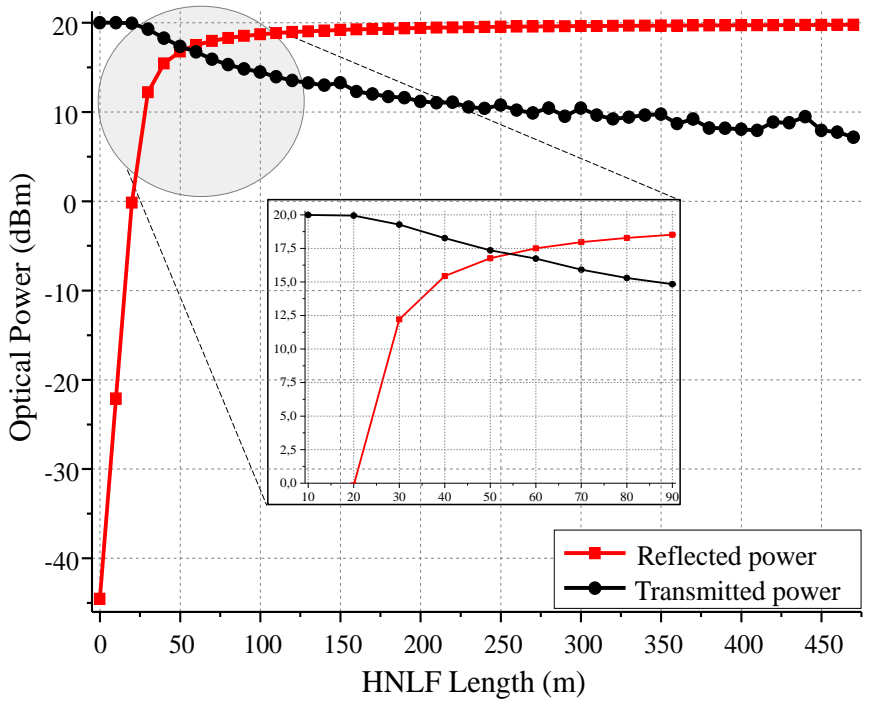

Fig. 7. Transmitted power and reflected power in the HNLF due to the SBS effect as a function of the HNLF length. 
Brillouin effect and still generating FWM products. It is worth mentioning the difficulty to make the fusion splicing between the HNLF and the SMF, due to the core size difference, i.e. 4 and $9 \mu \mathrm{m}$, respectively.

The performance investigation with the new HNLF length has been carried out using the same experimental setup from Fig. 4 for the new $35 \mathrm{~m}$ of HNLF piece. Fig. 8 depicts the transmitted and reflected power curves as a function of the optical input power for $35 \mathrm{~m}$ of HNLF. It is possible to verify a Brillouin threshold shifting from 15 to $29 \mathrm{dBm}$ when compared to Fig.5. The shifting allows the amplified RoF system to operate with power levels up to $29 \mathrm{dBm}$, without a significant impact from the SBS effect. Additionally, the intersection point between the transmission and reflection curves has not occurred due to the EDFA output power saturation, which occurs around $33 \mathrm{dBm}$.

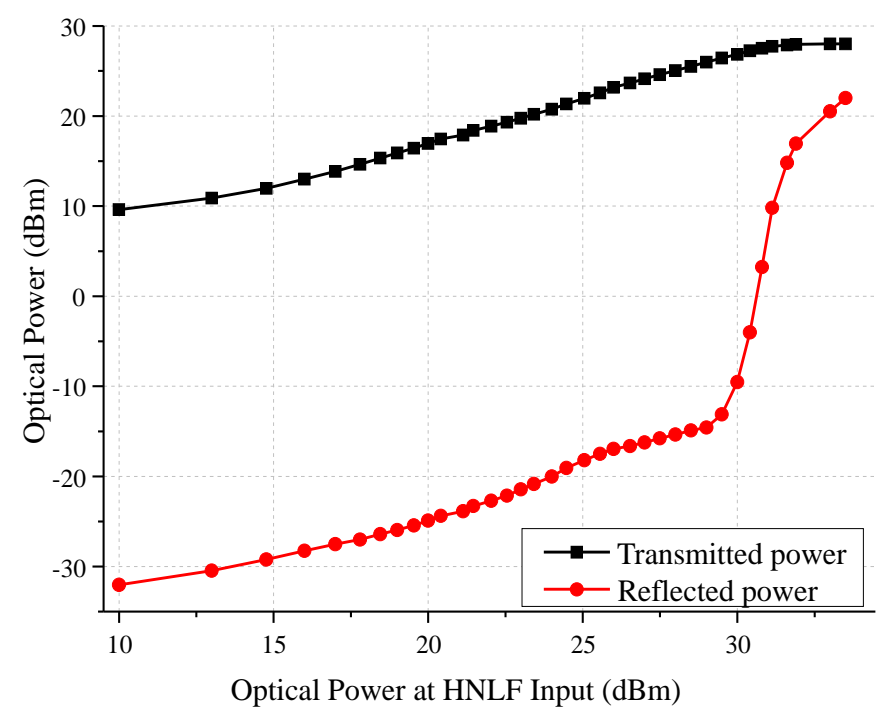

Fig. 8. Transmitted and reflected power levels as a function of the optical input power for $35 \mathrm{~m}$ of HNLF.

\section{B) Digital Analysis Using $35 \mathrm{~m}$ of HNLF}

As reported in Fig. 8, the $35 \mathrm{~m}$ of HNLF piece has shifted the Brillouin threshold to $29 \mathrm{dBm}$, enabling the photonic-assisted $\mathrm{RF}$ amplification system to operate at higher transmission power levels. As a consequence, the next step relied on evaluating the PAA performance using this shorter HNLF piece in terms of $\mathrm{EVM}_{\mathrm{RMS}}$ and as a function of the optical power for bandwidths up to $2 \mathrm{GHz}$. Fig. 9 presents a photography of the experimental setup used to the SBS characterization and PAA analysis, which is in accordance with the photonic-assisted amplification block diagram, presented in Fig. 2 (b).

Initially, the characterization consists of verifying the FWM generation, since the HNLF length was reduced and, consequently, the FWM efficiency and number of products could also be reduced. It is important to highlight only the first left or right FWM is selected for PAA approach, thus, highorder FWM products are unnecessary for the proposed photonically-amplified system. Fig. 10 exhibits the optical spectrum at the HNLF output, measured by an optical spectrum analyzer (OSA) with $0.07 \mathrm{~nm}$ resolution.

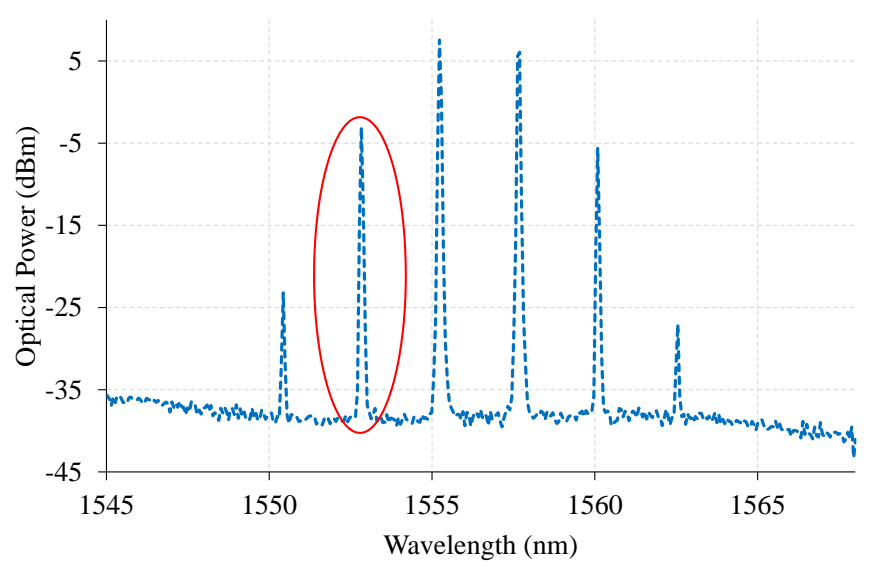

Fig. 10. Optical spectrum measured for $35 \mathrm{~m}$ of HNLF and the selected FWM product.

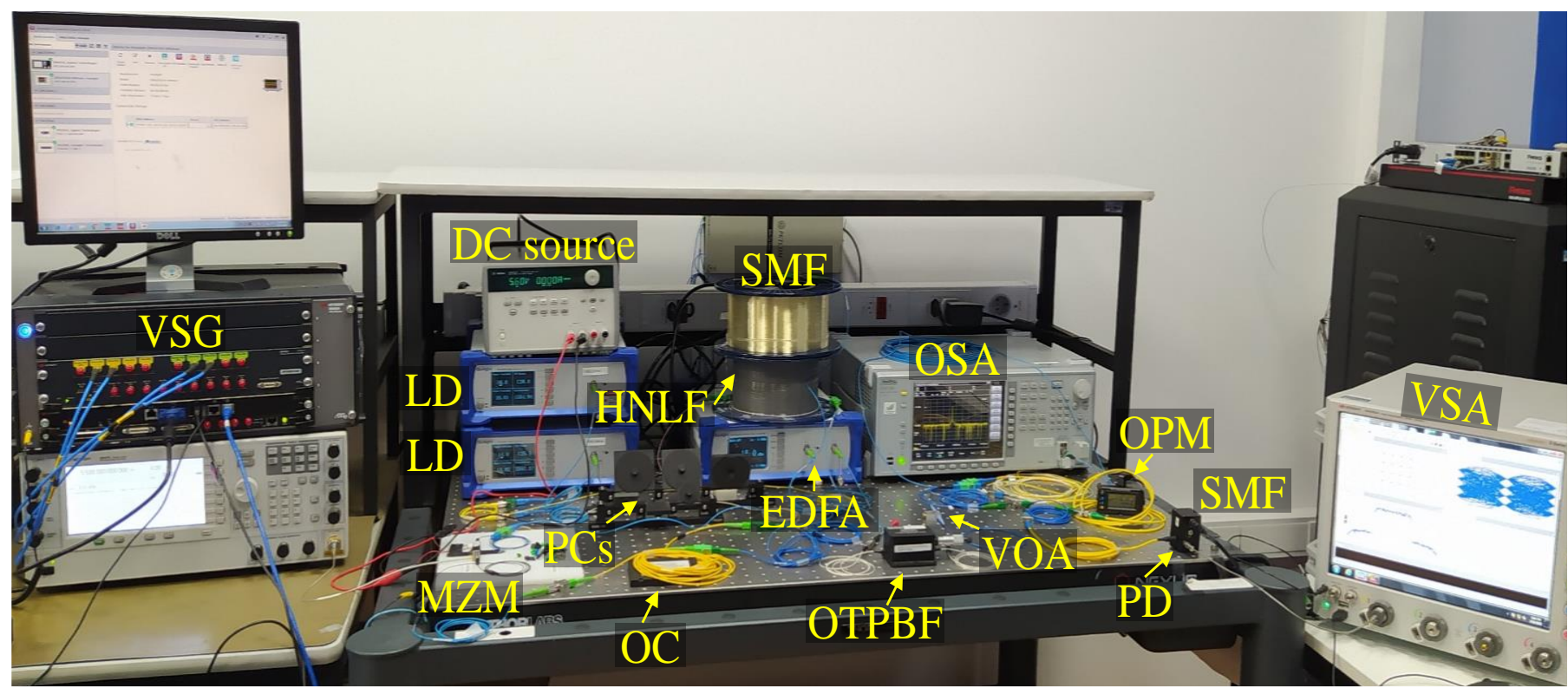

Fig. 9. Photography of the experimental setup for SBS characterization and PAA analysis. 
The EDFA output power has been properly set to $20 \mathrm{dBm}$, which is a typical power for the PAA technique [25]. One can observe the two laser wavelengths $(1,555.35$ and $1,557.65 \mathrm{~nm})$ and a considerable FWM products generation. The left FWM product has been selected due to its higher power level in comparison with the first right one. The optical power level difference is due to polarization conditions, since, the lasers have the same transmission power. Finally, the selected FWM product has been launched into a fronthaul composed of $12.5 \mathrm{~km}$ of SMF, reaching a photodetector.

The next step of the investigation consisted on evaluating the photonic-assisted RF amplification with the new piece of HNLF, regarding the 3GPP Release 15 requirements in terms of $\mathrm{EVM}_{\mathrm{RMS}}$. A vector signal generator from Keysight has been used to generate the RF signal with $500 \mathrm{MHz}$ bandwidth and roll-off factor equal to 0.15 , which corresponds to a $575 \mathrm{MHz}$ bandwidth allocation. The modulation order has been set to QPSK, 16-QAM and 64-QAM, reaching $1 \mathrm{Gbit} / \mathrm{s}, 2 \mathrm{Gbit} / \mathrm{s}$ and $4 \mathrm{Gbit} / \mathrm{s}$ total throughput, respectively. For comparison purposes with the results from Fig. 2 (c), we have realized the performance as a function of the optical input power for $35 \mathrm{~m}$ of HNLF, as presented in Fig. 11.

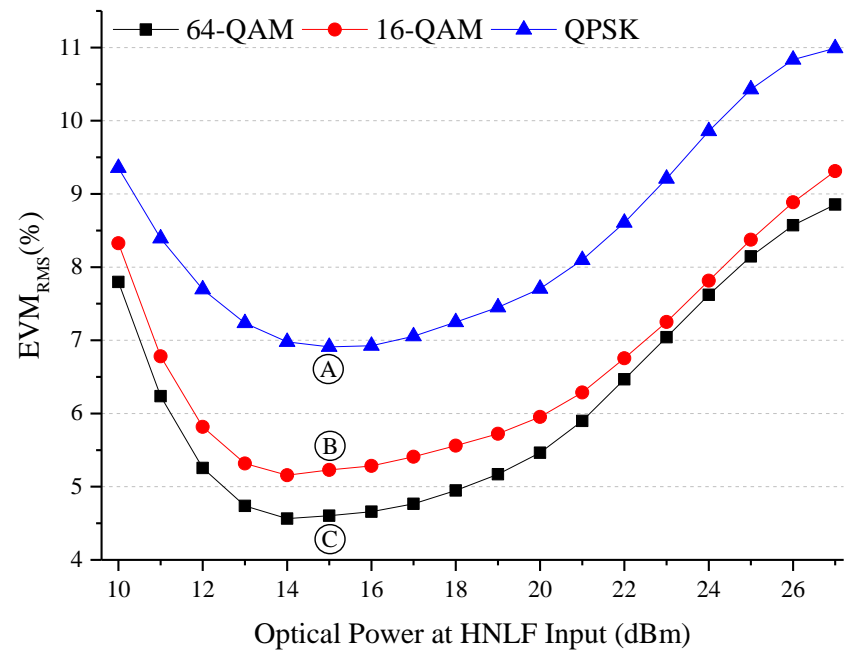

Fig. 11. EVM $\mathrm{EMS}_{\mathrm{R}}$ as a function of the optical power at the 35-meters HNLF input.

3GPP has standardized a maximum $\mathrm{EVM}_{\mathrm{RMS}}$ value for each modulation order, which meets the $5 \mathrm{G}$ systems specifications, namely [32][33]: $3.5 \%, 8.0 \%, 12.5 \%$ and $17.5 \%$ for $256-, 64-$, 16-QAM and QPSK, respectively. One can note the 3GPP recommendations have been achieved for all evaluated cases, except for 64-QAM with optical powers higher than $25 \mathrm{dBm}$. On the other hand, the results with $470 \mathrm{~m}$ (Fig. 2(c)) demonstrate much inferior digital performance. The QPSK modulation reaches the $3 \mathrm{GPP}$ EVMrMs limit at approximately $27 \mathrm{dBm}$ optical power, whereas the 16-QAM limit has been satisfied only from 17 to $23 \mathrm{dBm}$ optical power. The 64-QAM has not been reported, but a lower operating range is expected due to its lower $\mathrm{EVM}_{\mathrm{RMS}}$ limit. For $470 \mathrm{~m}$ of HNLF, the optimal operation point was around $20 \mathrm{dBm}$, whereas $35 \mathrm{~m}$ demanded $14 \mathrm{dBm}$. As a conclusion, the new and shorter piece of HNLF enhances the $\mathrm{EVM}_{\mathrm{RMS}}$ digital performance using $6 \mathrm{~dB}$ less optical power. Fig. 12 presents the correspondent constellations and eye diagrams at the marks labeled as "A", "B" and "C" from Fig. 11. The measured $\mathrm{EVM}_{\mathrm{RMS}}$ values were $6.95 \%$, $5.30 \%$ and $4.60 \%$ for the QPSK, 16-QAM, and 64-QAM modulations, respectively. The obtained values meet the requirements with margin, giving rise to well-defined constellation symbols and a clean eye diagram opening for all evaluated cases.
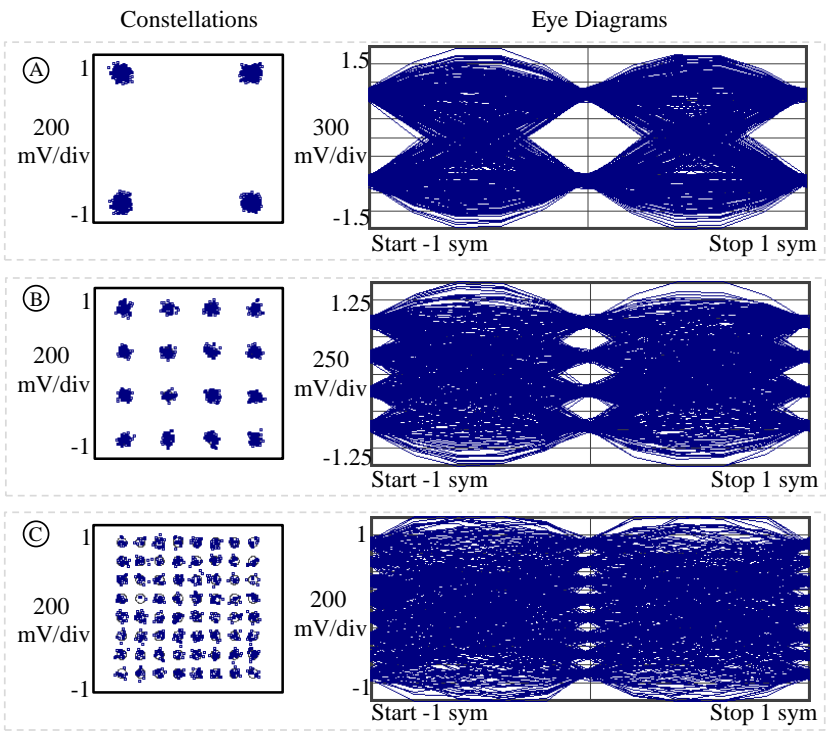

Fig. 12. Constellations and eye diagrams at the marks from Fig. 11.

The final step of the new HNLF length investigation has comprised the system throughput evaluation for higher bandwidths, as shown in Fig. 13. We have transmitted 16-, 64and 256-QAM modulation orders with bandwidths up to $2 \mathrm{GHz}$, which results in throughputs up to $16 \mathrm{Gbit} / \mathrm{s}$. The carrier frequency and electrical power have been set to $6 \mathrm{GHz}$ and $0 \mathrm{dBm}$, respectively. The investigation has aimed to measure the maximum data throughput for each modulation, considering the $3 \mathrm{GPP} \mathrm{EVM}_{\mathrm{RMS}}$ requirements, as outlined in Fig. 13. One can observe for all evaluated cases the $\mathrm{EVM}_{\mathrm{RMS}}$ values remained below the requirements attaining 8,12 and $16 \mathrm{Gbit} / \mathrm{s}$ for 16-, 64- and 256-QAM, respectively.

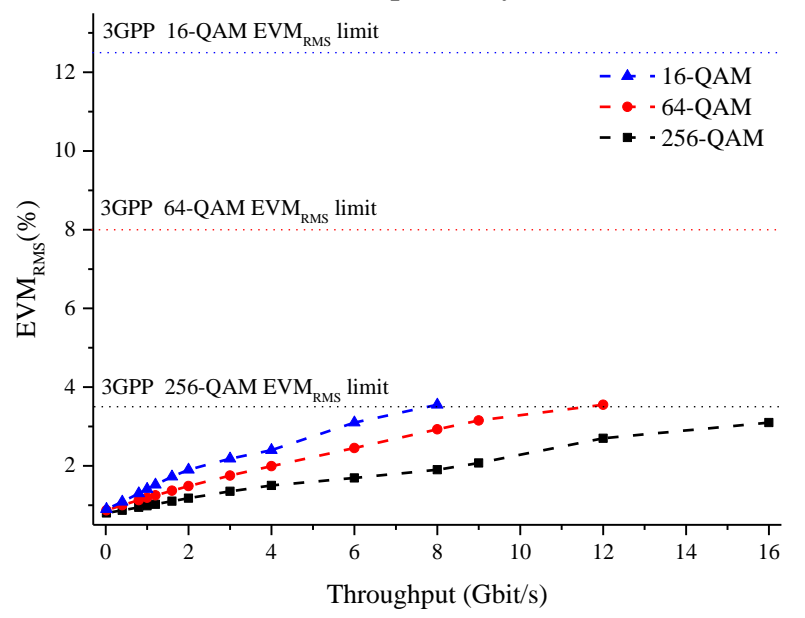

Fig. 13. Total throughput investigation as a function of $\mathbf{E V M}_{\mathrm{RMS}}$ for bandwidths up to $2 \mathrm{GHz}$. 


\section{CONCLUSIONS}

This paper has reported the Brillouin impact on photonicallyamplified radio over fiber systems. Experimental results have demonstrated reduced system power efficiency for optical power levels higher than $21 \mathrm{dBm}$ when using $470 \mathrm{~m}$ of HNLF due to stimulated Brillouin scattering. A heterodyne technique has been efficiently applied for detecting the presence of the nonlinear SBS effect in the PAA system. Therefore, we have been able to measure the Brillouin shift provoked by optical powers above the nonlinear effect threshold, which has resulted in an RF component at $9.1 \mathrm{GHz}$ carrier frequency. We have numerically investigated the optimum HNLF length for reducing the SBS effect. The intersections between the transmitted and reflected power at HNLF has occurred at $54 \mathrm{~m}$. Posterior, we have decided to use $35 \mathrm{~m}$ of HNLF piece for ensuring a trade-off between SBS effect reduction and FWM nonlinear efficiency. The $35 \mathrm{~m}$ of HNLF has ensured an increment of $14 \mathrm{~dB}$ on Brillouin threshold, allowing optical powers up to $29 \mathrm{dBm}$ at the HNLF input, without causing a significant impact on the system performance and still generating significant FWM products. Finally, the 35-m HNLF improved the $\mathrm{EVM}_{\mathrm{RMS}}$ digital performance and demanded $6 \mathrm{~dB}$ less optical power for attaining $19 \mathrm{~dB}$ photonics-assisted RF gain. Future works regard the application of this shorter HNLF piece in 5G fiber-wireless (FiWi) systems with multiple operating frequency bands, including $\mathrm{mm}$-waves.

\section{ACKNOWLEDGMENTS}

This work was partially supported by Rede Nacional de Ensino e Pesquisa (RNP), with resources from Ministério da Ciência, Tecnologia, Inovação e Comunicações (MCTIC), Grant No. 01250.075413/2018-04, under the Radiocommunication Reference Center (Centro de Referência em Radiocomunicações - CRR) project of Inatel. The authors thank the financial support from Coordenação de Aperfeiçoamento de Pessoal de Nível Superior (CAPES), Financiadora de Estudo e Projetos (FINEP), Fundação de Amparo à Pesquisa do Estado de Minas Gerais (FAPEMIG), Conselho Nacional de Desenvolvimento Científico e Tecnológico (CNPq) and Keysight Technologies. The authors also thank the technical support of Keysight Technologies and $\mathrm{Ph} . D$ Gustavo Silva Wiederhecker from instituto de física Gleb Wataghin (IFGW).

\section{REFERENCES}

[1] A. Osseiran, F. Boccardi, V. Braun, K. Kusume, P. Marsch, M. Maternia, O. Queseth, M. Schellmann, H. Schotten, H. Taoka, H. Tullberg, M. A. Uusitalo, B. Timus, and M. Fallgren, "Scenarios for $5 \mathrm{G}$ mobile and wireless communications: the vision of the METIS project," IEEE Communication Magazine., vol. 52, no. 5, pp. 26-35, May 2014, doi: 10.1109/MCOM.2014.6815890.

[2] ITU-R, "IMT vision - framework and overall objectives of the future development of IMT for 2020 and beyond," Recommendation ITU-R M.2083-0, September 2015.

[3] Qualcomm, "Making 5G NR a reality: leading the technology inventions for a unified, more capable $5 \mathrm{G}$ air interface," White paper, December 2016.

[4] I. Philbeck, "Connecting the unconnected: working together to achieve Connect 2020 Agenda targets," ITU White Paper, 2017.
[5] 3GPP, "5G; NR; User equipment (UE) radio transmission and reception; Part 3: Range 1 and Range 2 interworking operation with other radios," TS 38.101-3 version 15.3.0 Release 15, 2018.

[6] D. Zhang, M. Matthé, L. Mendes, and G. Fettweis, "A study on the link level performance of advanced multicarrier waveforms under MIMO wireless communication channels," IEEE Transactions on Wireless Communications., vol. 16, no. 4, pp. 2350-2365, April 2017, doi: 10.1109/TWC.2017.2664820.

[7] ETSI, "5G; Study on Scenarios and Requirements for Next Generation Access Technologies," TR 138913 V14.2.0, 2017.

[8] A. Tzanakaki, M. Anastasopoulos, I. Berberana, D. Syrivelis, P. Flegkas, T. Korakis, D. C. Mur, I. Demirkol, J. Gutierrez, E. Grass, Q. Wei, E. Pateromichelakis, N. Vucic, A. Fehske, M. Grieger, M. Eiselt, J. Bartelt, G. Fettweis, G. Lyberopoulos, E. theodoropoulou, and D. Simeonidou, "Wireless-optical network convergence: Enabling the 5G arquitecture to support operational and end-user services," IEEE Communication Magazine., vol. 55, no. 10, pp. 184-192, October 2017, doi: 10.1109/MCOM.2017.1600643.

[9] C. I, H. Li, J. Korhonen, J. Huang, and L. Han, "RAN revolution with NGFI (xhaul) for 5G," Journal of Lightwave Technology., vol. 36, no. 2, pp. 541-550, January 2018, doi: 10.1109/JLT.2017.2764924.

[10] C. I, J. Huang, R. Duan, C. Cui, J, Jiang, and L. Li, "Recent progress on C-RAN centralization and cloudification," IEEE Access, vol. 2, pp. 10301039, August 2014, doi: 10.1109/ACCESS.2014.2351411.

[11] J. Capmany and D. Novak, "Microwave photonics combine two worlds," Nature Photonics., vol. 1, no. 6, pp. 319-330, June 2007, doi: 10.1038/nphoton.2007.89

[12] J. Yao, "Microwave Photonics," Journal of Lightwave Technology., vol. 27, no. 3, pp. 314-335, February 2009, doi: 10.1109/JLT.2008.2009551.

[13] B. Skubic, G. Bottari, A. Rostami, F. Cavaliere, and P. Ohlen, "Rethinking optical transport to pave the way for $5 \mathrm{G}$ and the networked society," Journal of Lightwave Technology., vol. 33, no. 5, pp. 1084-1091, March 2015, doi: 10.1109/JLT.2015.2394297.

[14] H. R. D. Filgueiras, R.M. Borges, M. C. Melo, T.H. Brandão, and Arismar Cerqueira S. Jr., "Dual-band wireless fronthaul using a FSS-based focalpoint/Cassegrain antenna assisted by an optical midhaul," IEEE Access, vol. 7, pp. 112578-112587, August 2019, doi: 10.1109/ACCESS.2019.2934428.

[15] R. M. Borges, L. A. M. Pereira, H. R. D. Filgueiras, A. C. Ferreira, M. S. B. Cunha, E. R. Neto, D. H. Spadoti, L. L. Mendes, and Arismar Cerqueira S. Jr, "DSP-based flexible-waveform and multi-application $5 \mathrm{G}$ fiberwireless system," Journal of Lightwave Technology., vol. 38, no. 3, pp. 642-653, February 2020, doi: 10.1109/JLT.2019.2947916.

[16] G. Kalfas, C. Vagionas, A. Antonopoulos, E. Kartsakli, A. Mesodiakaki, S. Papaioannou, P. Maniotis, J. S. Vardakas, C. Verikoukis, and N. Pleros, "Next generation fiber-wireless fronthaul for 5G mmWave networks," IEEE Communication Magazine., vol. 57, no. 3, pp. 138-144, March 2019, doi: 10.1109/MCOM.2019.1800266.

[17] G. P. Agrawal, Nonlinear fiber optics: New York: Academic Press, 2001.

[18] B. Vidal, "Photonic millimeter-wave frequency multiplication based on cascaded four-wave mixing and polarization pulling," Optics Letters., vol. 37, no. 24, pp. 5055-5057, December 2012, doi: 10.1364/OL.37.005055

[19] S.A.S. Melo, A. R. do Nascimento, Arismar Cerqueira S. Jr., L.H.H. Carvalho, D.M. Pataca, J.C.R.F Oliveira, and H.L. Fragnito, "Frequency comb expansion based on optical feedback, highly nonlinear and erbiumdoped fibers', Optics Communication., vol. 312, pp. 287-291, February 2014, doi: 10.1016/j.optcom.2013.09.045.

[20] W. S. Wall and M. A. Foster., "Ultra-wideband gain in microwave photonic links using four-wave mixing," Conference on Lasers and Electro-optics (CLEO), San Jose, USA, 2012, doi: 10.1364/CLEO_SI.2012.CTu3A.4

[21] Arismar Cerqueira S. Jr. N. Cañas-Estrada, D. F. Noque, R. M. Borges, S.A.S. Melo, N. G. González, and J. C. R. F. Oliveira, "Photonic-assisted microwave amplification using four-wave mixing," IET Optoelectronics., vol. 10, no. 5, pp. 163-168, September 2016, doi: 10.1049/ietopt.2014.0173

[22] A. L. M. Muniz, R. M. Borges, Regivan N. Da Silva, D. F. Noque, and Arismar Cerqueira S. Jr., "Ultra-broadband photonics-based RF front-end toward 5G networks," Journal of Optical Communications and Networking ., vol. 8, no. 11, pp. B35-B42, November 2016, doi: $10.1364 / \mathrm{JOCN} .8 .000 \mathrm{~B} 35$. 
[23] A. L. M. Muniz, D. F. Noque, R. M. Borges, A. Bogoni, M. Hirano, and Arismar Cerqueira S. Jr., "All-optical RF amplification towards Gbps communications and millimeter-waves applications," Microwave and Optical Technology Letters ., vol. 59, no. 9, pp. 2185-2189, 2017, doi: 10.1002/mop.30704.

[24] D. F. Noque, R. M. Borges, A. L. M. Muniz, A. Bogoni, and Arismar Cerqueira S. Jr., "Thermal and dynamic range characterization of a photonics-based RF amplifier," Optics Communication., vol. 414, pp. 191-194, May 2018, doi: 10.1016/j.optcom.2018.01.015.

[25] E. S. Lima, R. M. Borges, L. A. Melo Pereira, H. R. Dias Filgueiras, A. M. Alberti and A. Cerqueira Sodré, "Multiband and Photonically Amplified Fiber-Wireless Xhaul," in IEEE Access, vol. 8, pp. 4438144390, 2020, doi: 10.1109/ACCESS.2020.2977562.

[26] M. Takahashi, J. Hiroishi, M. Tadakuma, and T. Yagi, "Improvement of FWM conversion efficiency by SBS-suppressed highly nonlinear dispersion-decreasing fiber with a strain distribution," in European Conference on Optical Communication., (ECOC) Brussels, Belgium, 2008, doi: 10.1109/ECOC.2008.4729208.

[27] F. Willems, W. Muys, and J. Leong, "Simultaneous suppression of stimulated Brillouin scattering and interferometric noise in externally modulated lightwave AM-SCM systems," IEEE Photonics Technology Letters., vol. 6, no. 12, pp. 1476-1478, December 1994, doi: $10.1109 / 68.392206$.

[28] K. Shiraki, M. Ohashi, and M. Tateda, "Suppression of stimulated Brillouin scattering in a fibre by changing the core radius," Electronics Letters, vol. 31, no. 8, pp. 668-669, April 1995, doi: 10.1049/el:19950418.

[29] E. S. Lima, L. A. M. Pereira, R. M. Borges and Arismar Cerqueira S. Jr., "Impact of Brillouin effect in optical-wireless systems with RF amplification in the optical domain [In Portugues]", in Simpósio Brasileiro de Telecomunicações e Processamento de Sinais (SBrT), Petrópolis, Brazil, 2019.

[30] R. Y. Chiao, C. H. Townes, and B. P. Stoicheff, "Stimulated Brillouin Scattering and Coherent Generation of Intense Hypersonic Waves," Physical Review Letters, vol. 12, no. 21, pp. 592-595, May 1964, doi: 10.1103/PhysRevLett.12.592.

[31] Hui, Rongqing, and Maurice O'Sullivan. Fiber optic measurement techniques. Academic Press, 2009.

[32] 3GPP, "Group Radio Access Network; NR; User Equipment (UE) radio transmission and reception; Part 1: Range 1 Standalone," TS 38.101-1 version 15.5.0 Release 15, 2019.

[33] 3GPP TS 38.104, "5G; NR; Base Station (BS) radio transmission and reception", v. 15.2.0, 2018.

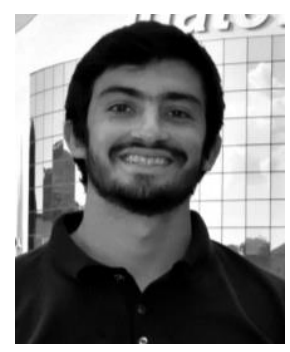

Eduardo Saia Lima received his B.Sc. and M.Sc. degrees from Inatel, Brazil, in 2017 and 2019, respectively and is a Ph.D student in Telecommunication in the same institution. Has experience in teaching for being a tutor in electronic circuits, digital electronics and optics communications on undergraduate courses from Inatel from 2017 to 2019 in the Teaching Internship Program (PED). Currently acts as researcher for the Wireless and Optical Convergent Access (WOCA) Laboratory at Inatel.

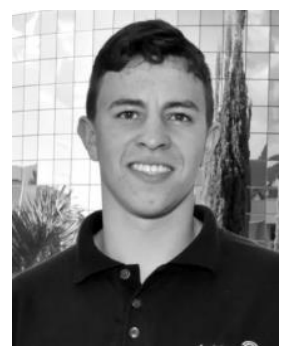

Luiz Augusto Melo Pereira received the B.Sc. degree in telecommunication engineering and the M.Sc. degree in telecommunications from the National Institute of Telecommunications (Inatel), Brazil, in 2017 and 2020, respectively. He is currently a Ph.D. student in telecommunications at Inatel and integrates the Lab. WOCA (Wireless and Optical Convergent Access) research team. His research interests include optical communications, wireless systems, and microwave photonics.

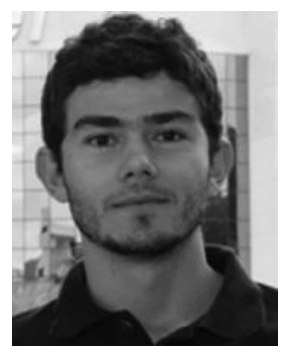

Ramon Maia Borges received the B.Sc. degree in electrical engineering and the M.Sc. degree in telecommunications from the National Institute of Telecommunications (Inatel), Brazil, in 2012 and 2015, respectively. $\mathrm{He}$ is currently working toward the Ph.D. degree in electrical engineering at the Federal University of Itajubá (UNIFEI), Brazil. He is currently a Researcher for the Radiocommunications Reference Center Project at Inatel. His research interests include optical communications, wireless systems, and microwave photonics.

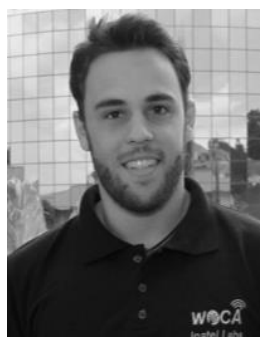

Matheus Sêda Borsato Cunha received the B.Sc. degree in electrical engineering and the M.Sc. degree in telecommunications from the National Institute of Telecommunications (Inatel), Brazil, in 2015 and 2018, respectively. He is currently working toward the Ph.D. degree in electrical engineering at the Federal University of Itajuba, Brazil. He is a Researcher with the Wireless and Optical Convergent Access Laboratory, Inatel. His research interests include integrated optics, microwave photonics, optoelectronic systems, optical communications, and optical-wireless networks.

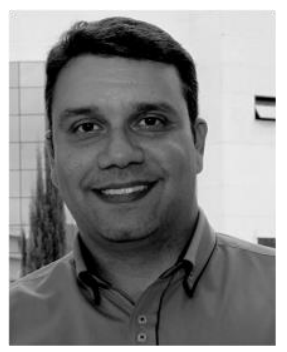

Arismar Cerqueira Sodré Junior received the B.Sc. degree in electrical engineering from the Federal University of Bahia, Brazil, in 2001, the M.Sc. degree from the State University of Campinas (Unicamp), Brazil, in 2002, and the Ph.D. degree from Scuola Superiore Sant'Anna, Italy, in 2006. He was an Invited Researcher and Professor from many world-recognized universities, such as the University of Oulu (2017), Scuola Superiore Sant'Anna, Italy (2015 and 2017), Danish Technical University, Denmark (2013), Max-Planck Institute, Germany (2010), and University of Bath, U.K. (2004, 2005, and 2007). He is an Associate Professor of the National Institute of Telecommunications, Brazil, to work in the same position. Since 2009, he has been acting as a Coordinator of R\&D Projects on diverse areas of telecommunications, including antennas, 5G networks, radars and microwave photonics. He is a holder of 10 patents, has transferred 20 products to the industry, and has published 244 scientific papers. 